\title{
La especialidad de cirugía general, ¿una especie en extinción?
} Is the specialty of general surgery in extinction?

\author{
Erich O.P. Basurto-Kuba* \\ Hospital General de México, Ciudad de México, México
}

La cirugía ha acompañado al ser humano en el transcurso de su historia, aunque inicialmente solo para resolver algunas patologías de manera cruenta (heridas, abscesos, fracturas, etc.).

La primera división en la medicina ocurre cuando aparece la cirugía como actividad independiente, al inicio mal vista, con los llamados cirujanos barberos, considerados poco científicos, que estaban relegados a procedimientos dolorosos, sin anestesia y con muy frecuente desarrollo de infecciones, por lo que tenían muy mala fama entre la comunidad. Sin embargo, a partir de la aparición de la anestesia y la antisepsia, que se conjuntaron a finales del siglo XIX y principios del siglo $x x$, se empezó a desarrollar una verdadera especialidad, la cirugía, que avanzó de tal forma que se puso a la par de la medicina interna e incluso la rebasó en proyección y prestigio. Surgieron los grandes cirujanos en el mundo occidental, de la talla de Billroth, Kocher, Bassini y Halsted, por mencionar solo algunos entre muchos otros; y en nuestro país Montes de Oca, Lavista, Urrutia, etc. Tal fue el avance de la cirugía que a principios del siglo xx se le otorgó el premio Nobel a un cirujano, el Dr. Emil Theodor Kocher, por sus trabajos en cirugía de tiroides desarrollando una técnica de tiroidectomía estandarizada que disminuyó de manera importante la morbilidad y la mortalidad quirúrgica y así mismo por el reconocimiento de los efectos sobre la fisiología de los pacientes sometidos a tiroidectomía.

Estos cirujanos impusieron las reglas del manejo de los pacientes y diseñaron y desarrollaron técnicas quirúrgicas, muchas de las cuales se utilizan hasta nuestros días, a veces solo modificadas por el uso de la tecnología actual (cirugía laparoscópica, engrapadoras, fuentes de energía, etc.), pero que siguen manteniendo los mismos principios. Los cirujanos de esa época eran cirujanos generales en el sentido estricto de la palabra; tenían acceso a todas las partes de la economía humana que pudieran ser abordadas quirúrgicamente, aunque ya empezaban a aparecer alguna subdivisiones, de las cuales las iniciales fueron la ginecoobstetricia y la ortopedia.

No solo se mejoró la atención de los pacientes por los cirujanos o se desarrollaron nuevas técnicas e instrumental, sino que además, en el ámbito de la educación quirúrgica, se empezó a dejar la forma tutorial de enseñar y se iniciaron las primeras escuelas quirúrgicas con un programa bien establecido, con el objeto de formar cirujanos generales muy bien entrenados que pudieran replicar los modelos de atención y enseñanza en otras ciudades; y que tenía como base que el cirujano en entrenamiento viviera en el hospital; de ahí nace el concepto de residencia en cirugía general, en nuestro país inicialmente impulsada en los años 1940 en el Hospital General de México por el Dr. Aquilino Villanueva. Este sistema estaba basado en la competencia piramidal, creándose el puesto de jefe de residentes, que lo ganaba el médico residente más destacado. En ese momento, los cirujanos generales egresados de una residencia formal eran los médicos más prestigiosos y respetados.

Con la evolución explosiva del conocimiento médico y el desarrollo vertiginoso de la tecnología en la medicina, se fue haciendo necesario que los cirujanos empezaran a dedicarse con más profundidad a tratar de manera individual las diferentes partes de la economía humana para poder dominarla. Así empezaron a aparecer las subespecialidades 0

Correspondencia:

${ }^{\star}$ Erich Otto Paul Basurto-Kuba

Agrarismo, 208-701

Col. Escandon, Del. Miguel Hidalgo

C.P. 11800, Ciudad de México, México

E-mail: ebasurto_kuba@ hotmail.com
Fecha de recibido:17-05-2018

Fecha de aceptado: 20-05-2018

DOI://dx.doi.org/10.24875/CIRU.M18000046
Cir Cir. 2018;86:305-307

Contents available at PubMed www.cirugiaycirujanos.com 
superespecialidades, dejando algunos cirujanos por completo el resto del ámbito de la cirugía general y haciéndose superespecialistas en solo una de las disciplinas de la cirugía general. Así aparecieron la neurocirugía, la cirugía oncológica, la cirugía cardiovascular, la cirugía coloproctológica, etc., Paulatinamente se ha ido pulverizando el ámbito del cirujano general, quien fue perdiendo campo de acción en los hospitales donde se cuenta con todas las subespecialidades, al grado de que en muchos hospitales certificados y de prestigio nacional ya no sale el residente como cirujano general, sino como cirujano de tubo digestivo en el mejor de los casos.

Tanto se fue perdiendo el campo de acción que ya es difícil saber qué es o qué hace un cirujano general, tanto para la misma comunidad médica como mucho más para la sociedad en general, que lo confunde con un médico general y no lo identifica como especialista.

La Asociación Mexicana de Cirugía General define al cirujano general como un médico titulado que cursa 4 años de entrenamiento en cirugía general en un hospital con reconocimiento y presenta un examen universitario al final de su especialidad y un examen de certificación por el Consejo Mexicano de Cirugía General; de esta manera puede ejercer la especialidad obteniendo la Cédula de Especialista en Cirugía General que otorga la Dirección General de Profesiones de la Secretaría de Educación Pública (SEP).

Los hospitales ofrecen la especialidad de cirugía general a la comunidad de médicos aspirantes a una residencia y los reconocidos por la Universidad Nacional Autónoma de México (UNAM) siguen el programa llamado PUEM (Plan Único de Especialidades Médicas)', que en lo que concierne a la cirugía general enumera una serie de áreas que deben incluirse en la enseñanza teórica y práctica de la cirugía general, como son la cirugía de tubo digestivo desde el esófago hasta el ano, el hígado y las vías biliares, el páncreas, la patología ginecológica y la cesárea, la mama, la patología maligna y benigna de todas ellas, algunas patologías de cirugía de tórax, vascular y urológica, por mencionar solo algunas. Dicho programa es muy ambicioso y desgraciadamente en muy pocas sedes se logra cumplir tanto la parte teórica como mucho más difícil la práctica; es así que, cuando se quiere exponer a los residentes a las diferentes patologías, estos deben rotar por otras sedes.

El Consejo Mexicano de Cirugía General incluye en su examen de certificación todos los temas contenidos en el PUEM y por lo tanto, si se quiere obtener el certificado, debe tenerse el conocimiento de todos estos; es frecuente ver sustentantes en el examen que confiesan no haber visto nunca algunos tipos de patología quirúrgica sobre los que se les examina.

Lo anterior paradójicamente choca con las políticas institucionales de algunos hospitales en donde no solo no se cumple completamente con el PUEM, sino que incluso las autoridades hospitalarias prohíben que el servicio de cirugía general tenga injerencia en algunos tipos de patología que supuestamente se le deben enseñar al residente para cumplir su entrenamiento, lo que resulta en la formación de cirujanos generales limitados.

Una vez que el residente termina su entrenamiento se ve en dos escenarios: el de trabajar como cirujano general o el de continuar su entrenamiento y desarrollar una superespecialidad. Dadas las condiciones actuales en las ciudades con hospitales de tercer nivel, en donde el cirujano general es menospreciado y muy limitado en su actuar, la mayoría de los residentes prefieren continuar con su entrenamiento, pero cuando el flamante cirujano general empieza a trabajar en una comunidad donde no existe atención de tercer nivel se ve en la obligación de atender pacientes con problemas diversos, algunos de los cuales no vio durante su residencia, lo que puede redundar en tratamientos y cirugías no seguras.

Esto no es privativo de nuestro país, pues está ocurriendo en todo el mundo y es frecuente ver foros internacionales y publicaciones en los que se aborda el tema de la enseñanza y del futuro de la cirugía general. Aquí transcribo lo que mencionaron Boyarsky, et al. ${ }^{2}$ en 1995 en un artículo que publicaron en The American Surgeon en relación a la cirugía general en los EE.UU.: «El futuro de cualquier especialidad en medicina depende en gran parte de la elección que hacen los candidatos; se obtuvieron datos de los jefes de residentes de cirugía general que terminaron en 1990 y se compararon su actitudes con las de un estudio similar realizado en 1985 y la respuesta fue que los residentes de cirugía general están escogiendo cada vez más subespecialidades, ya que no perciben que el ser cirujano general sea suficiente. Esto tiene amplias implicaciones para el futuro de la cirugía general como especialidad que requiere cambios significativos en nuestros programas de residencia». 
Debemos hacernos una serie de preguntas: ¿Se debe seguir ofreciendo la especialidad de cirugía general en los hospitales? ¿Debe continuar vigente el PUEM de cirugía general tal como está diseñado a la fecha o debe adecuarse a la época actual? ¿Qué tipo de residentes de cirugía general queremos formar? ¿Qué tipo de cirujano general necesita el país?

Creo que la cirugía general no debe desaparecer, simplemente porque el $80 \%$ de la patología quirúrgica en el país es resuelta por los cirujanos generales y solo un pequeño porcentaje de pacientes son atendidos en los hospitales que cuentan con clínicas de alta especialidad. Esto nos debe hacer ver que debemos ofrecer un entrenamiento más amplio a nuestros residentes.

\section{Bibliografía}

1. Plan Único de Especializaciones Médicas (PUEM) en Cirugía General. Facultad de Medicina, UNAM, División de estudios de posgrado, Subdivisión de especializaciones médicas, Coordinación de desarrollo curricular. México, D.F. 2009 Plan de estudios aprobado por el Consejo Universitario el 21 de abril de 1994. Coordinación editorial del Dr. Bernardo Pintos Aguilar. Ciudad de México, junio de 2016.

2. Boyarsky AH, Donetz A, Greco RS. The future of general surgery characterized by general surgical chief residents. Am Surg. 1995;61:244-51. 